\title{
THE OBSERVATION OF MONOCHROMATIC INTENSITY \\ DISTRIBUTIONS IN PLANETARY NEBULAE \\ BY MEANS OF ELECTRONOGRAPHY*
}

\author{
MERLE F. WALKer \\ and \\ Gerald E. KRON \\ (Lick Observatory, University of \\ California, U.S.A.) \\ (U.S. Naval Observatory, U.S.A.)
}

A BSTRACT

The linear response and high information gain of the electronographic process make it an important new technique for the observation of monochromatic intensity distributions in planetary nebulae. Preliminary results for NGC 6720, obtained with the U.S. Navy electronic camera and the 61-inch astrometric reflector, are presented.

Work in recent years (Frieser and Klein, 1958; Frieser et al., 1959; Vernier, 1959; Méallet, 1961 ; Duchesne and Méallet, 1962; Valentine, 1966) has demonstrated that a linear relationship exists in electronography between the number of incident electrons and the photographic specular density in the silver-halide recording emulsion, to very high values of the density (Kron and Papiashvili, 1967; Kron, 1967; Walker, 1967). Emulsions exposed in an electronographic-type image-converter (electronic camera) will thus have a photographic density proportional to the intensity of the light incident on the photocathode over a large range of intensities. In addition, the fine grain and high quantum efficiency of the electronographic process permits the recording of the same information in $\leqslant 1 / 15$ the time required photographically, depending upon the wavelength (Walker, 1966b).

Electronic cameras have been used in astronomical spectrophotometry (see e.g. Walker and Popper, 1964; Aller and Walker, 1965; Walker, 1966a), in measuring the surface brightnesses of galaxies (Kron, 1967) and globular clusters (Kron and Papiashvili, 1967), and it has been shown that they can be used for the determination of stellar magnitudes (Walker and Kron, 1967). However, the small sizes of the photocathodes of presently available electronic cameras restrict their application to studies of objects or fields of relatively small extent.

One problem for which present electronic cameras are well suited is that of studying monochromatic intensity distributions in planetary nebulae. Using the electronic camera, direct-intensity pictures can be made of the nebulae in the light of particular emission lines by observing through narrow-band interference filters. Equal-interval

* Contributions from the Lick Observatory, No. 269.

Osterbrock and O'Dell (eds.), Planetary Nebulae, 282-286. (O) I.A.U. 
iso-intensity contour maps of the distribution of intensity or surface-brightness in the nebulae may then be made from these pictures by scanning them in a Joyce, Loebl recording microdensitometer, operated as an isodensitracer. This microdensitometer has been carefully designed to record specular densities up to a photographic density of 6 . The action of the densitometer depends ultimately on matching the plate transmission with that of an optical wedge. The wedges supplied by the manufacturer have a linear variation in density with distance along their length to an accuracy of $\pm 1 \%$. Contours corresponding to (equal) density increments on the plate as small as 0.005 may be traced. Contours of successively higher density (or intensity) are indicated by the three different modes of operation of the recording pen, in the following sequence: blank, dash, dot.

A preliminary study of this technique has been made using the U.S. Navy Electronic Camera, developed by one of us (Kron), attached to the focus of the 61 -inch astrometric reflector of the U.S. Naval Observatory Flagstaff Station. The scale in the focal plane of this telescope is $13^{\prime \prime} .5 / \mathrm{mm}$, which is reduced electronically to $21.1 / \mathrm{mm}$ on the recording emulsion in the electronic camera. The telescope is geometrically $f / 10$, but owing to the large size of the flat secondary mirror, operates optically at $f / 12$. Tests indicate that despite the slow optical speed of the telescope, images of planetary nebulae of moderate surface brightness can be obtained in the light of the stronger emission lines with reasonable exposure times. Thus, using a telescope having an optical speed of about $f / 5$, it should be possible to obtain direct-intensity pictures of a number of planetaries in the light of relatively faint high- and low-excitation lines.

Figure 1 reproduces the electronographic picture obtained in the light of $\lambda 4861$ $\mathrm{H} \beta$, while other pictures were also obtained in $\lambda 3727-29$ [OII] and $\lambda 5007$ [OIII]. The scale of the reproduction is $1.1 / \mathrm{mm}$, and the exposure times and filter data for all the pictures are given in Table 1.

Table 1

Filter and exposure data for electronographic observations of NGC 6720

Filter

$\begin{array}{lccc}\lambda & \begin{array}{c}\text { Half-Intensity } \\ \text { Width }(\AA)\end{array} & \begin{array}{c}\text { Peak } \\ \text { Transmission }(\%)\end{array} & \begin{array}{c}\text { Exposure } \\ \text { Time }\end{array} \\ 3727-29 & & & \\ 4861 & 40 & 42 & 1^{\mathrm{h}} 00^{\mathrm{m}} \\ 5007 & 19 & 56 & 131 \\ & 21 & 66 & 030\end{array}$

The sensitivity of the (Sb-Cs) cathode of the electronic camera was about $20 \mu \mathrm{a} / \mathrm{lu}$, and the images were recorded on Ilford K5 nuclear research emulsion. The plates were developed $5 \mathrm{~min}$ in $\mathrm{D}-19$ at $65^{\circ} \mathrm{F}$. The slightly granular appearance of the pictures results from the fact that the image tube used was an experimental one 


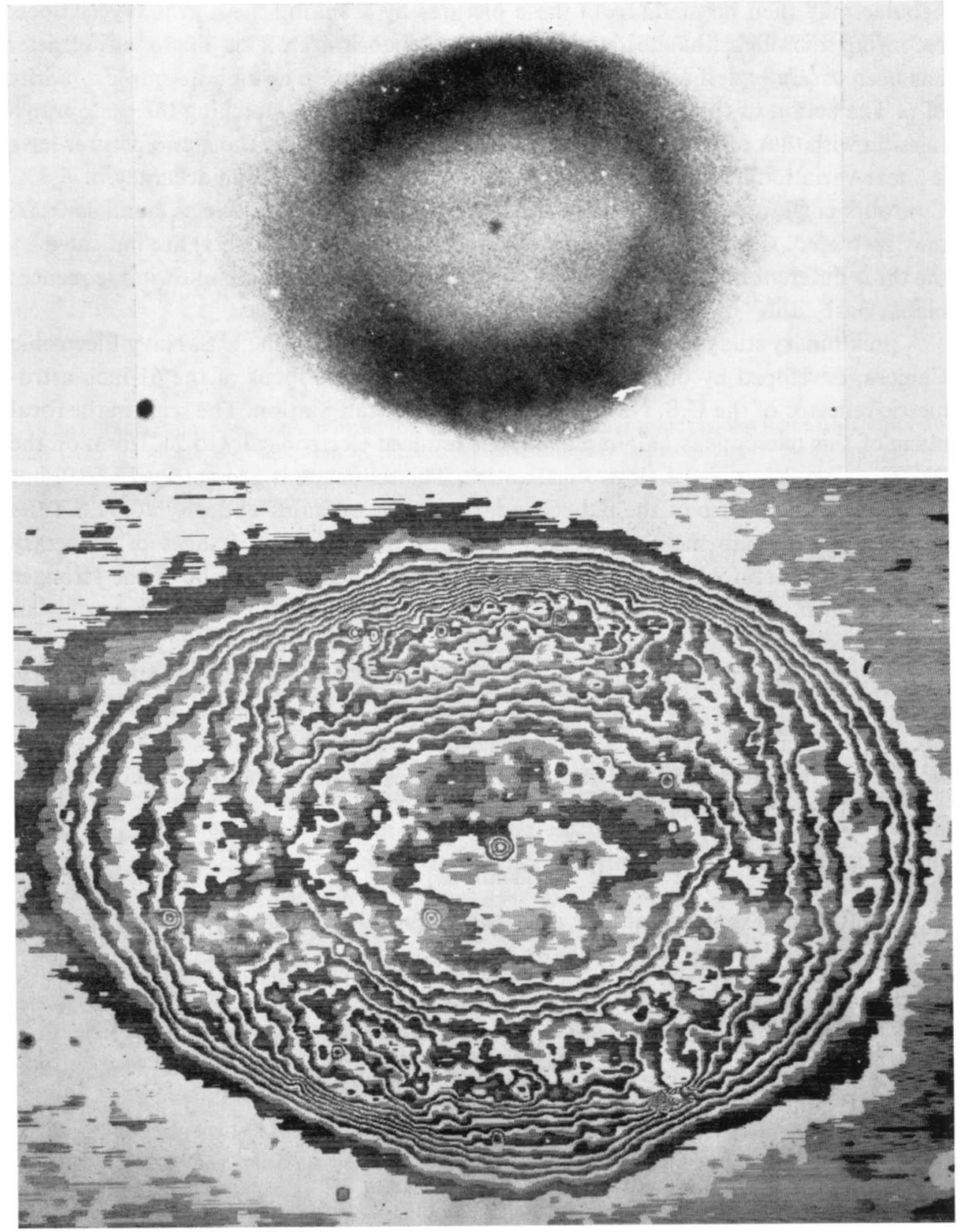

FIG. 1. Upper: Electronographic picture of NGC 6720 in the light of $\lambda 4861 \mathrm{H} \beta$; lower: Intensity distribution of NGC 6720 in the light of $\lambda 4861 \mathrm{H \beta}$. 
with a ground cathode surface to eliminate the effects of light reflected to the photocathode from the elements of the electron-optical system. The resolution of the pictures, however, is set by the seeing at the times of observation; the best seeing occurred during the exposure on $\lambda 4861$, where the image of the central star has a diameter of 1 ."8.

The lower part of Figure 1 shows the isodensity or iso-intensity tracing obtained from the picture taken in $\mathrm{H} \beta$ above. In this tracing the size of the scanning aperture, projected on the emulsion, was $50 \mu \times 50 \mu$, the interval between successive scans was $5 \mu$, and the density increment between successive scans was 0.02 . The enlargement of the contour map over the original plate is 27.8 times. Comparing these iso-intensity contours with those published by Aller (1956), the large increase in information recorded by the electronographic method is apparent. It is to be noted that these particular Ilford plates contained a number of defects. These are readily identifiable on the contour maps by comparing these with the original pictures. Eventually, the two-dimensional maps of a given nebula in different emission lines might be put on a homogeneous intensity system for that nebula by means of the electronographic spectrophotometry of the planetary nebulae recently carried out by Aller and Walker $(1965,1967)$, which gives the relative intensities of the emission lines at a few particular points in the nebula. Such material could then be used for two-dimensional spectrophotometric investigations such as the study of the detailed ionization structure of the nebula.

The present results indicate that the application of electronography to the photometric study of the planetary nebulae constitutes an important new observational technique. It is also clear that in order to extract the maximum amount of information by this method, the electronographic pictures must be taken under conditions of excellent seeing, and with a telescope giving sufficient scale that the definition of the pictures is always seeing-limited.

\section{Acknowledgements}

It is a pleasure to thank Mr. K. Janes for his assistance in making both the observations and the contour maps of NGC 6720. Travel support for one of us (Walker) to visit Flagstaff was provided by a grant from the National Science Foundation.

\section{References}

Aller, L.H. (1956) Gaseous Nebulae, Chapman and Hall, London, p. 247.

Aller, L.H., Walker, M.F. (1965) Astrophys. J., 141, 1318.

Aller, L.H., Walker, M.F. (1967) in preparation.

Duchesne, M., Méallet, M. (1962) C.r. Soc. sav. Paris, 254, 1400.

Frieser, H., Klein, E. (1958) Z. angew. Phys., 10, 337.

Frieser, H., Klein, E., Zeitler, E. (1959) Z. angew. Phys., 11, 190.

Kron, G.E. (1967) unpublished. 
Kron, G. E., Papiashvili, I.I. (1967) Publ. astr. Soc. Pacific, 79, 9.

Méallet, M. (1961) Mémoire présenté à la Faculté des Sciences de l'Université de Paris pour obtenir le Diplôme d'Études Supérieures de Sciences Physiques.

Valentine, R. (1966) Advances in Microscopy, 1, 180.

Vernier, P. (1959) Bull. astr., Paris, 22, 83.

Walker, M.F. (1966a) in Stellar Evolution, Ed. by R.F. Stein and A. G.W. Cameron, Plenum Press, New York, p. 405.

Walker, M.F. (1966b) Advances Electronics, Electron Phys. 22B, 761.

Walker, M.F. (1967) unpublished.

Walker, M. F., Kron, G.E. (1967) Publ. astr. Soc. Pacific, 79, 551.

Walker, M. F., Popper, D. M. (1964) Astrophys. J., 139, 168. 